\title{
Numerical Investigation of Graphene-Gold SPR- Based Biosensor
}

\author{
Saeideh Jafari ${ }^{1}$, Jafar Mostafavi Amjad ${ }^{2}$, Ramin Mohammadkhani ${ }^{1}$ and Peyman Jahanshahi ${ }^{3 *}$ \\ ${ }^{1}$ Department of physics, Faculty of Science, University of Zanjan, 45371-38791, Iran \\ ${ }^{2}$ Department of Physics, Institute for Advanced Studies in Basic Sciences (IASBS), Iran \\ ${ }^{3}$ Institute of Intelligent Manufacturing and Information Engineering, School of Mechanical Engineering, Shanghai Jiao Tong University, \\ China
}

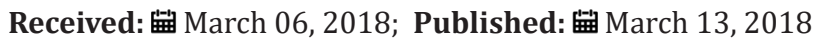

*Corresponding author: Peyman Jahanshahi, School of Mechanical Engineering, Shanghai Jiao Tong University, China, Email: peyman840@gmail.com

\begin{abstract}
This short study is aimed to investigate the graphene-gold surface plasmon resonance (SPR)-based biosensor by optimizing the graphene and gold layers respectively. Gold $(\mathrm{Au})$ is used as a noble-metal material whereas graphene is used to avoid Au from oxidation and enhance the reflectance intensity of biosensor as well as increase molecular absorbance on the sensor surface for improving sensitivity. Numerical analysis is performed using finite-element method (FEM) by comparing the sensing performance such as reflectance intensity that explains the sensor sensitivity. The measurements are presented with various graphene thin film thicknesses in $3 \mathrm{~nm}, 5 \mathrm{~nm}$, and $6 \mathrm{~nm}$ with $800 \mathrm{~nm}$ operating wavelength, and then considered the different gold thicknesses $(10 \mathrm{~nm}$ to $70 \mathrm{~nm})$. The proposed sensor shows that the five-layer graphene-40nm thick gold SPR-based sensor gave better performance with narrower plasmonic spectrum line width. The obtained results also indicate that the graphene-gold SPR-based sensor is suitable for medical diagnostics in immunoassays.
\end{abstract}

Keywords: Surface Plasmon Resonance; Biosensor; Graphene; Numerical Analysis

Abbreviations: SPR: Surface Plasmon Resonance; Au: Gold; Ti: Titanium; FEM: Finite-Element Method

\section{Introduction}

Sensitive detections of diseases - which relevant antigens, antibodies, or proteins were critical for many biomedical studies and diagnostics [1-3]. The assays based on SPR technique here increased dramatically and the number of reporting researchers has been quickly growing [4-6]. The technique of SPR is very sensitive to a refractive index variation in sensing medium. This variation occurs due to binding of analyte (in a liquid sample) to their affinity ligands (immobilized on the chip surface) when the liquid sample comes in contact to the sensor surface. Therefore, material which is in contact with the liquid sample (top layer of sensor), terms of the refractive index and the absorption of molecules, is very influential and important $[7,8]$. In the present study, to enhance the sensitivity of the SPR sensor for biomolecules, a number of graphene layers are generated onto an optimized gold thin film. To see the points of proposed structure, the numerical analysis is carried out for different graphene-gold SPR-based configurations. The most advantages of the use graphene layers can be pointed to more efficient biomolecular absorbance compared with gold, passivation of the sensor surface against oxidation, highly hydrophilic layered material, and be able to control of the SPR response by manipulating the number of coated graphene layers [9-12]. Due to the specific nature of graphene, the refractive index of this material in the visible range was estimated to be $1 . \mathrm{n}=3+\mathrm{iC} / 3$, where is the wavelength in and the constant $C$ equals $5.446 \mu m^{-1}$.

Moreover, the thickness of the graphene layer can be obtained as $\operatorname{tg}=0.34 \mathrm{~nm} \times \mathrm{N}$, where $\mathrm{N}$ is the number of graphene layers [13]. As noted in Table 1, the proposed SPR structure (Figure 1) is numerically analysed in the specified values of materials' refractive index and thickness at a free space wavelength of $800 \mathrm{~nm}$. In the first step, the graphene thickness is considered (three, five, and six) and applied one by one in each analysis. As shown in Figure $2 \mathrm{a}$, the reflected light is more close to zero with acceptable narrow 
line width at five-layer graphene. Therefore, it is fixed the graphene layers and continued the study for finding an optimized thickness of gold. For this purpose, the gold thickness is swept from $10 \mathrm{~nm}$ to $70 \mathrm{~nm}$ in the presence of five layers of graphene. Figure $2 \mathrm{~b}$ clearly shows the reflection is best resulted in $40 \mathrm{~nm}$ gold thickness, whether in excellent subsidence and narrowing of its width.

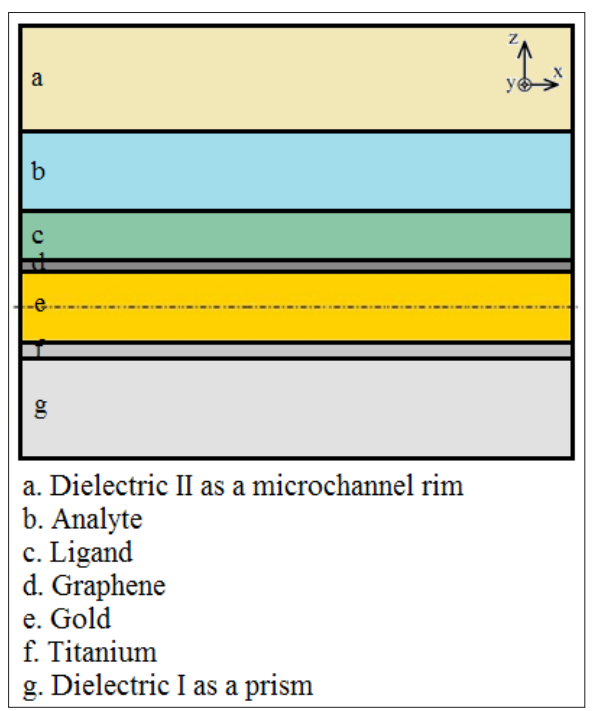

Figure 1: The SPR structure, dielectric II-gold-dielectric I waveguide structure.

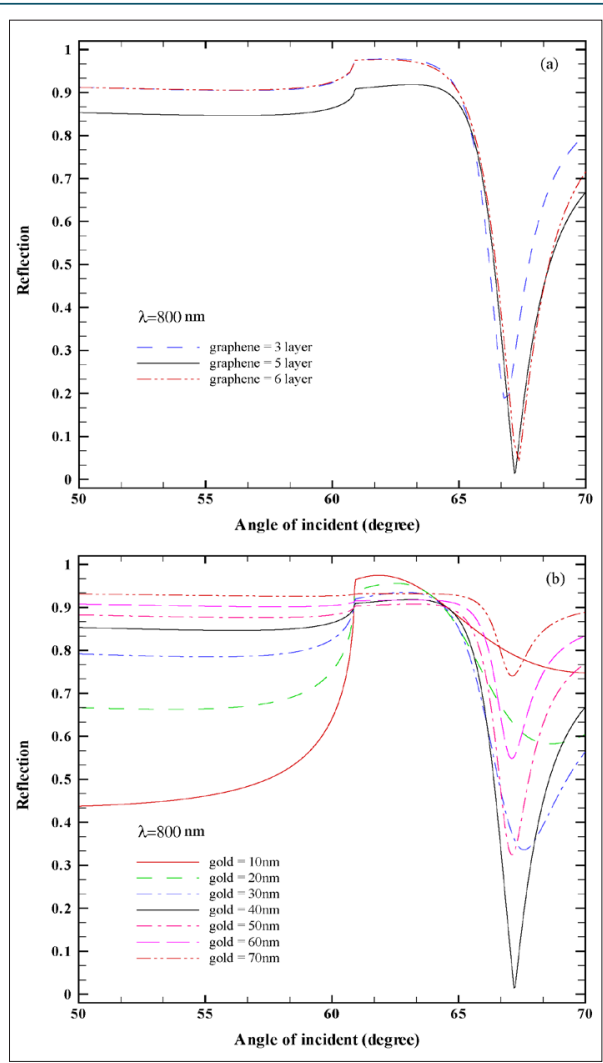

Figure 2: Reflectance against incident angle of 50 to 70 degree (a) with different graphene layers (gold thickness $40 \mathrm{~nm}$ ) and (b) with different gold layers (5 layers graphene), at $800 \mathrm{~nm}$.
Table 1: The values of materials refractive index and thickness at a free space wavelength of $800 \mathrm{~nm}$.

\begin{tabular}{|c|c|c|}
\hline Material & Refractive Index (RI) & Thickness (nm) \\
\hline Microchannel rim & 1.52 & 1000 \\
\hline Analyte & 1.329 & 1250 \\
\hline Ligand & 1.333 & 100 \\
\hline Graphene & $\begin{array}{c}3+\mathrm{i}(5.446[\mu \mathrm{m}-1] / 3) \\
\lambda\end{array}$ & $\begin{array}{c}0.34 \times \mathrm{N}(\mathrm{N}=3,5, \\
\text { and } 6)\end{array}$ \\
\hline Gold & $0.16+\mathrm{i} 4.84$ & $10-70$ \\
\hline Titanium & $3.14+\mathrm{i} 4.01$ & 2 \\
\hline Prism & 1.51 & 1000 \\
\hline
\end{tabular}

\section{Conclusion}

Performance of graphene and gold thin film thicknesses in our proposed configuration has been numerically investigated. It was found that the optimal number of graphene layer (fivelayer) based on the reflectance intensity which is proportional to sensor performance enhancement. For better functionality of our structure, the thickness of gold layer was investigated as well and best chosen in value of $40 \mathrm{~nm}$. Since the graphene acts as the biorecognition element so the proposed graphene-gold SPR-based biosensor provides a promising potential for using in medical rapid diagnostic approaches and intelligent biomedical manufacturing systems.

\section{Acknowledgement}

This work has been supported by National Science Foundation of China (No. 51435009).

\section{References}

1. P Jahanshahi, Q Wei, Z Jie, M Ghomeishi, SD Sekaran, et al. (2017) Kinetic analysis of IgM monoclonal antibodies for determination of dengue sample concentration using SPR technique Bioengineered 8(3): 239247.

2. P Jahanshahi, SD Sekaran, FRM Adikan (2015) Optical and analytical investigations on dengue virus rapid diagnostic test for IgM antibody detection. Medical \& Biology Engineering \& Computing 53(8): 1-9.

3. P Jahanshahi, E Zalnezhad, SD Sekaran, FRM Adikan (2014) Rapid Immunoglobulin M-Based Dengue Diagnostic Test Using Surface Plasmon Resonance Biosensor. Scientific Reports 4(3851).

4. P Jahanshahi, A Parvizi, FR Mahamd Adikan (2013) Three-dimensional modeling of surface plasmon resonance based biosensor 8801: 880109 .

5. KN Shushama, MM Rana, R Inum, MB Hossain (2017) Graphene coated fiber optic surface plasmon resonance biosensor for the DNA hybridization detection: Simulation analysis. Optics Communications 383: 186-190.

6. K Patel, S Halevi, P Melman, J Schwartz, S Cai, et al. (2017) A Novel Surface Plasmon Resonance Biosensor for the Rapid Detection of Botulinum Neurotoxins. Biosensors 7(3): 32.

7. J Homola (2008) Surface Plasmon Resonance Sensors for Detection of Chemical and Biological Species. Chemical Reviews 108(2): 462-493.

8. I Abdulhalim, M Zourob, A Lakhtakia (2008) Surface Plasmon Resonance for Biosensing: A Mini-Review. Electromagnetics 28(3): 214-242. 
9. P Subramanian, Adam Lesniewski, Izabela Kaminska, Alexis Vlandas, Alina Vasilescu, et al. (2013) Lysozyme detection on aptamer functionalized graphene-coated SPR interfaces. Biosensors and Bioelectronics 50C: 239-243.

10. MM Giangregorio, M Losurdo, GV Bianco, E Dilonardo, P Capezzuto, et al. (2012) Synthesis and characterization of plasmon resonant gold nanoparticles and graphene for photovoltaics. Materials Science and Engineering: B 178(9): 559-567.

11. DC Elias, RR Nair, TMG Mohiuddin, SV Morozov, P Blake, et al. (2009) Control of graphene's properties by reversible hydrogenation: evidence for graphane. Science 323(5914): 610-613.

12. DW Boukhvalov, MI Katsnelson (2008) Chemical functionalization of graphene with defects. Nano Letters 8(12): 4373-4379.

13. M Bruna, S Borini (2009) Optical constants of graphene layers in the visible range. Applied Physics Letters 94(3): 666.
(C) Commons Attribution 4.0 License

To Submit Your Article Click Here:

Submit Article

DOI: $10.32474 /$ OAJBEB.2018.01.000120

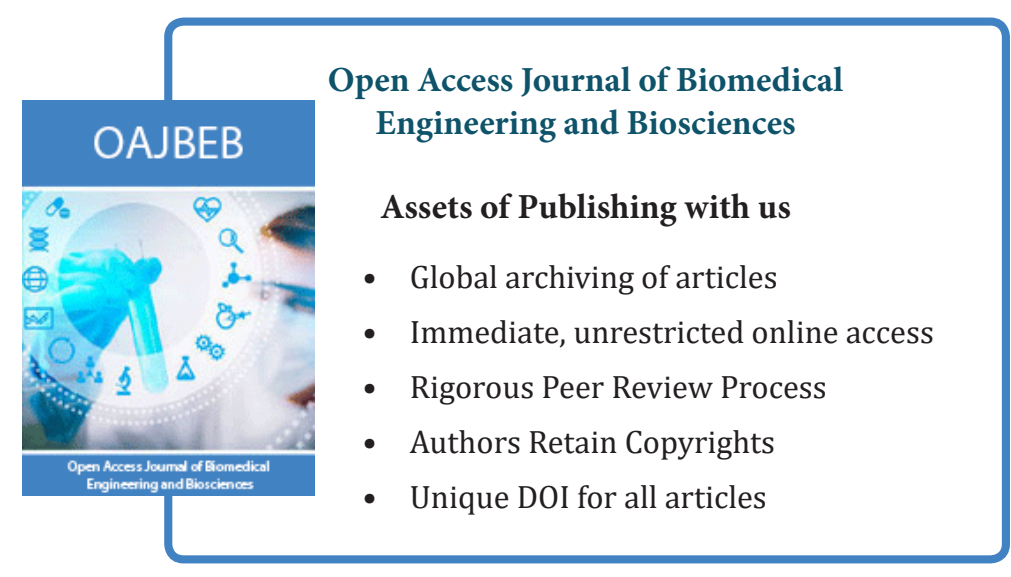

